EPJ Web of Conferences 32, 02007 (2012)

DOI: $10.1051 /$ epjconf/20123202007

(C) Owned by the authors, published by EDP Sciences, 2012

\title{
Influence of high energy electrons on ECRH in LHD
}

\author{
S. Kubo ${ }^{1,2, a}$, H. Takahashi ${ }^{1}$, T. Shimozuma ${ }^{1}$, Y. Yoshimura ${ }^{1}$, M. Nishiura ${ }^{1}$, H. Igami ${ }^{1}$, S. Ogasawara $^{2}$, \\ and R. Makino ${ }^{2}$ \\ 1 National Institute for Fusion Science, Toki 509-5292, Japan \\ 2 Department of Energy Engineering and Science, Nagoya University, Nagoya 464-8603, Japan
}

\begin{abstract}
The central bulk electron temperature of more than $20 \mathrm{keV}$ is achieved in LHD as a result of increasing the injection power and the lowering the electron density near $2 \times 10^{18} \mathrm{~m}^{-3}$. Such collision-less regime is important from the aspect of the neoclassical transport and also the potential structure formation. The presences of appreciable amount of high energy electrons are indicated from hard X-ray PHA, and the discrepancy between the stored energy and kinetic energy estimated from Thomson scattering. ECE spectrum are also sensitive to the presence of high energy electrons and discussed by solving the radiation transfer equation. The ECRH power absorption to the bulk and the high energy electrons are dramatically affected by the acceleration and the confinement of high energy electrons. The heating mechanisms and the acceleration process of high energy electrons are discussed by comparing the experimental results and the ray tracing calculation under assumed various density and mean energy of high energy electrons.
\end{abstract}

\section{Introduction}

Recent upgrade of the ECRH system in LHD[1] enabled to study the plasma confinement properties at the far low collisional or collision-less regime in the helical system where specific confinement features are predicted from the neo-classical transport theory. These specific features includes confinement degradation in the so called " $1 / v$ " regime where the ripple loss become dominant but radial electric field can drastically alter the situation[2]. In order to discuss the confinement properties in such collision-less regime, the accurate estimation of the behavior of the high energy electrons as well as that of the bulk electron temperature is required, since the high energy electrons can absorb injected ECRH power at the relativistically down shifted frequency and can deposit their energy to the bulk electrons out of the region where they absorb the energy from injected EC wave. Thus, the power deposition profile which is the key parameter in the transport analysis can be much affected by the presence of high energy electrons. One of the most reliable and well established methods to experimentally deduce the power deposition profile is using the transient analysis of the thermal electron cyclotron emission (ECE)[3]. In case of low density, high energy electrons are easily created under high power ECRH and the ECE can be non-thermal. The emission spectrum can not indicate local bulk electron temperature any more. In other words, such non-thermal ECE include integrated distribution in the real and velocity space [4-6].

It is also known that the presence of high energy electrons can alter the ECRH absorption, mainly due to the finite Larmor effect and the relativistic effect. In an inhomogeneous magnetic field configuration, the relativistic down shift of the cyclotron resonance causes the shift in the absorption region and also the appearance of the higher harmonic resonances for high energy electrons. The ECRH heating scenario in such low collisional plasma has to be explored taking the relativistic effect into account.

\footnotetext{
a e-mail: kubo@lhd.nifs.ac.jp
} 


\section{Achievement of high electron temperature and production of high energy electrons}

Using upgraded ECRH (of more than 3MW injection power), the plasma parameter region of the low density and high temperature is explored. With the careful adjustment of the magnetic field and the focal point, the central bulk electron temperature exceeded $20 \mathrm{keV}$ at the center. This bulk electron temperature is derived by averaging over several shots of YAG-Thomson scattering data. The YAGThomson scattering system in LHD gives reliable bulk electron temperature up to $20 \mathrm{keV}$, since it adopts blue shift side filters. It is confirmed that these estimations of the bulk electron temperature are not affected by the presence of high energy electrons discussed hereafter.

High temperature low density plasma produced by ECRH normally contains appreciable fraction of high energy electrons. In Fig. 1 are over plotted the time evolutions of the plasma parameters for high $n_{\mathrm{e}}\left(1.2 \times 10^{19} \mathrm{~m}^{-3}\right)$ low $\left(0.2 \times 10^{19} \mathrm{~m}^{-3}\right)$ and very low $\left(0.1 \times 10^{19} \mathrm{~m}^{-3}\right)$ density shots $($ as indicated in the column b) )with identical ECRH injection pattern as shown in a). It should be noted that the diamagnetic stored energy (Fig.1c) for the low and very low density cases are almost comparable up to $3.8 \mathrm{~s}$ even though the difference in the density is about factor 3. Furthermore, the stored energy for the very low density case gradually approaches to that for high density case toward the end of the shot. These stored energy behaviors are well explained by the production and acceleration of high energy electrons. The presence and acceleration of the high energy electrons are confirmed by the Hard X ray pulse height analysis. The results show that the energy reaches to $100 \mathrm{keV}$ in the very low density case.

In Fig.1d) are shown the time evolutions of the central electron temperature measured by YAGThomson scattering for these three cases. The central bulk electron temperature exceeds far above $10 \mathrm{keV}$ for the low density case, but stays below $10 \mathrm{keV}$ for both high and very low density cases.

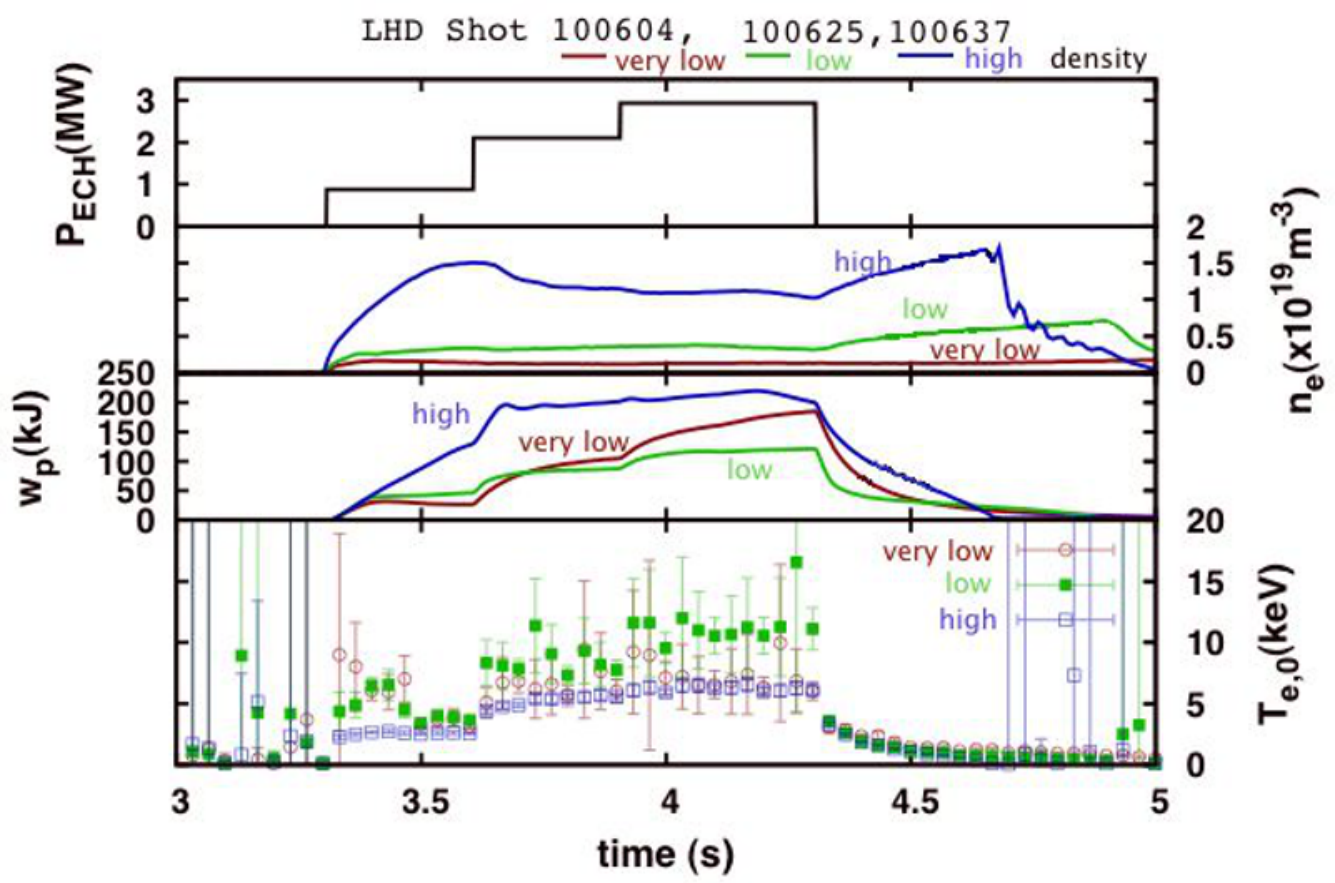

Fig. 1. Time evolutions of a) ECRH injection power, b) electron density, c) stored energy and d) central electron temperature for high $\left(1.2 \times 10^{19} \mathrm{~m}^{-3}\right)$ low $\left(0.2 \times 10^{19} \mathrm{~m}^{-3}\right)$ and very low $\left(0.1 \times 10^{19} \mathrm{~m}^{-3}\right)$ density shots. 


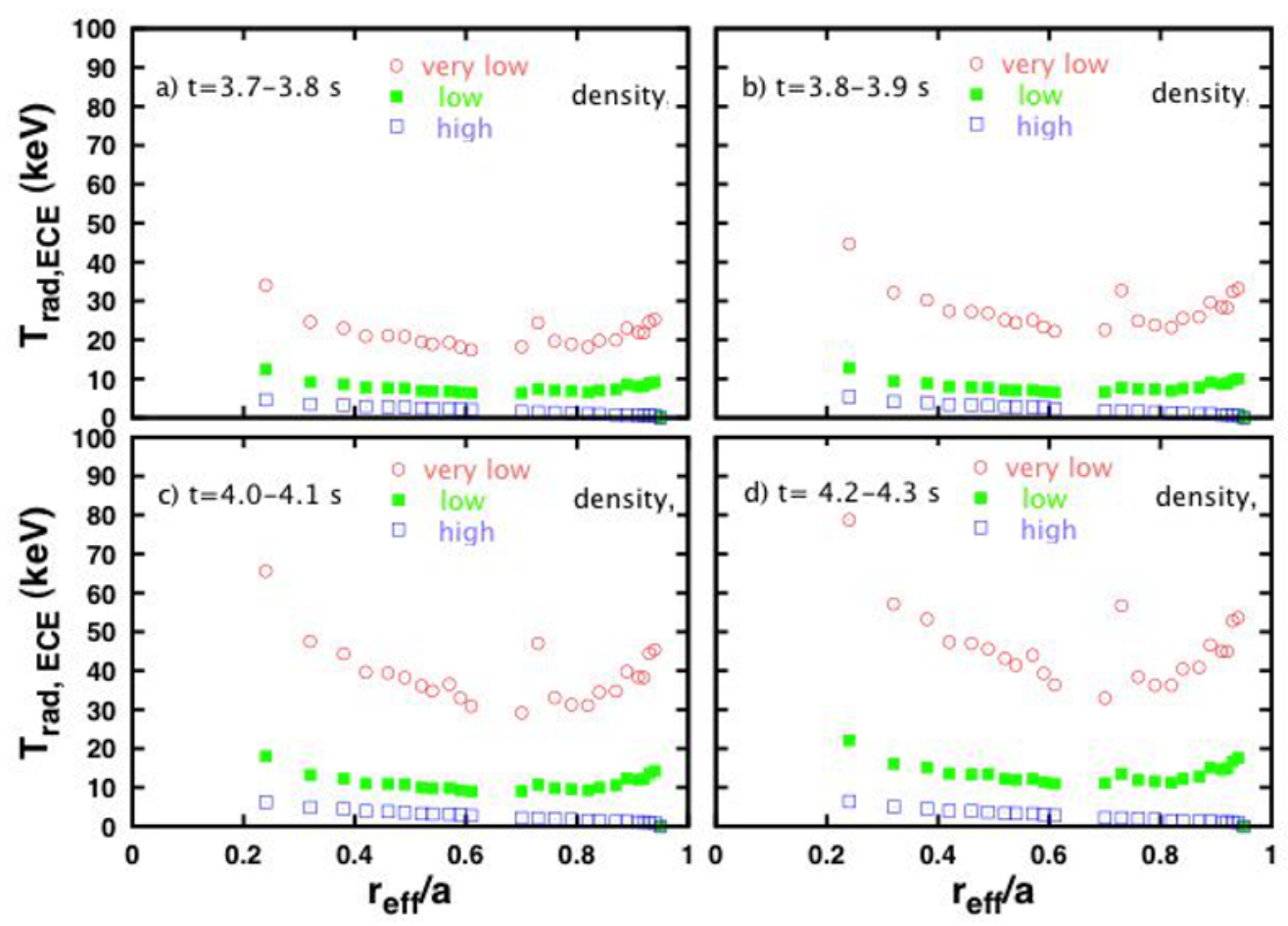

Fig. 2. ECE spectra plotted as a function of averaged minor radius corresponding to a second harmonic resonance position for the time slice at a) $t=3.7-3.8 \mathrm{~s}, \mathrm{~b}$ ) $\mathrm{t}=3.8-3.9 \mathrm{~s}$, c) $\mathrm{t}=4.0-4.1 \mathrm{~s}$ and d) $\mathrm{t}=4.2-4.3 \mathrm{~s}$ of each shot shown in Fig.1.

This decrease in the central electron temperature of very low density case can not be explained by the decrease in the absorption ratio depending on the density, since the one path absorption is almost 100 $\%$ even in the very low $n_{\mathrm{e}}$ of $0.1 \times 10^{19} \mathrm{~m}^{-2}$ at $10 \mathrm{keV}$. These results suggest that the power reached to the bulk electrons at the center decreased for the very low density case as compared with low density case.

The electron cyclotron emission non-thermal features are also consistent with the energy range and the density of high energy electrons as shown in Fig.2. Figure 2 show ECE intensity plotted as a function of corresponding cold second resonance position in $\operatorname{LHD}(144.5 \mathrm{GHz}-110.5 \mathrm{GHz})$. Intensity is indicated as ECE radiation temperature. Open square, closed square and open circle corresponds the case for the density of 1.2, 0.3 and $0.1 \times 10^{19} \mathrm{~m}^{-3}$ in Fig. 2, respectively. ECE intensity is averaged over a) $\mathrm{t}=3.7-3.8 \mathrm{~s}$, b) $3.8-3.9 \mathrm{~s}$, c) $4.0-4.1 \mathrm{~s}$ and d) $4.2-4.3 \mathrm{~s}$. It should be noted that the maximum scale of each figure is $100 \mathrm{keV}$ and only the high density case corresponds to the thermal electron temperature. The ECE intensity at high frequency side are ascribed to the non-thermal feature of the high energy electrons of relativistically down shifted emission from the core region. While those at low frequency side are ascribed to the down shifted second or third harmonic emission at optically thin region in the plasma periphery.

\section{Effects of high energy electrons on the ECRH power deposition}

Fig.2 suggests that the high energy electrons exist not only in the central region of the plasma but also peripheral region. That is also expected from the difference in the drift surface of the high energy electrons from that of bulk electrons[5]. 

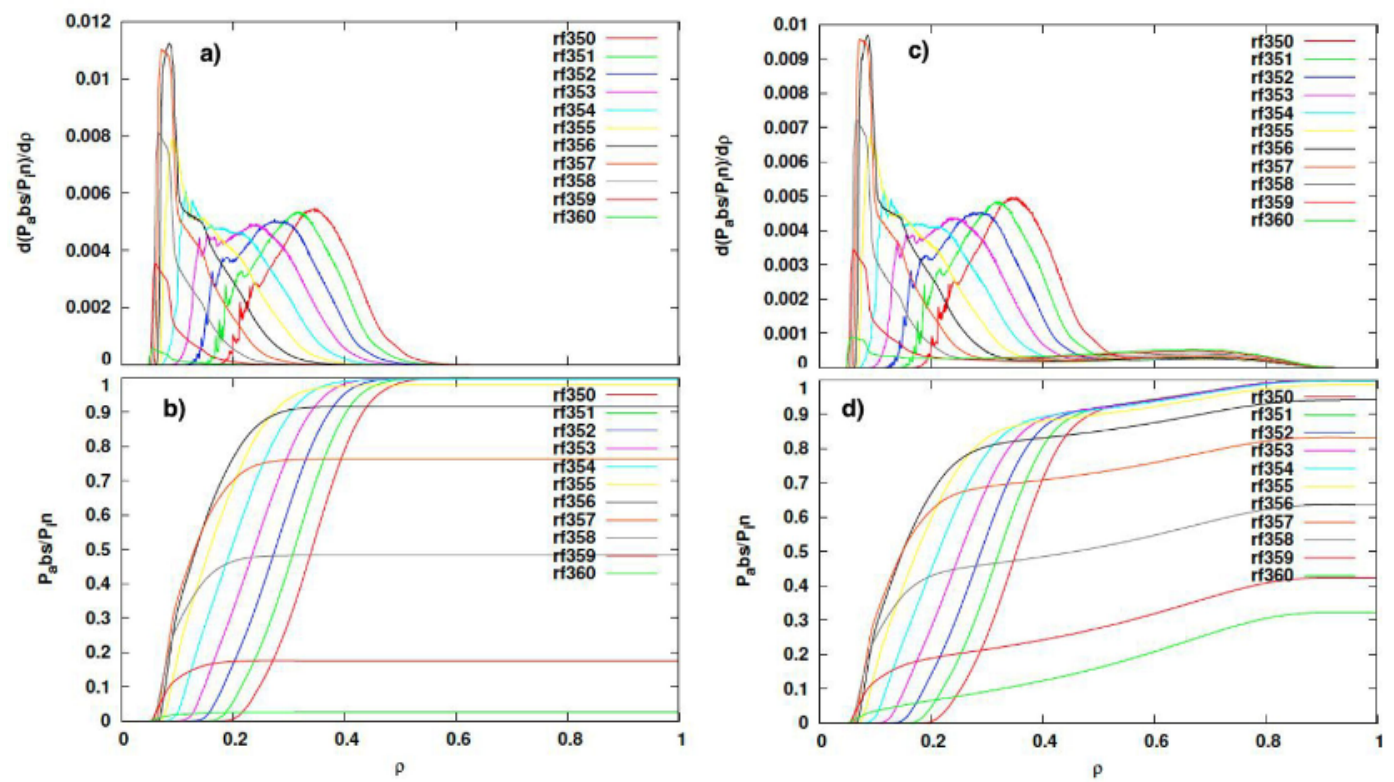

Fig. 3. An example of raytrace calculation results a),b) without and c), d) with the high energy contribution. a),c) indicate power deposition profile and b), d) are the integrated power absorption rate as a function of averaged minor radius for various focal points. $8 \%$ of the total electrons out of bulk electrons with $T_{\text {e,bulk }}(\rho)=15^{*}\left(1-\rho^{2}\right)^{2}$ $\mathrm{keV}$ Maxwellian are replaced by electrons with $T_{\mathrm{e}, \mathrm{high}}(\rho)=100 *\left(1-\rho^{2}\right)^{2} \mathrm{keV}$ Maxwellian. Total electron density is set $n_{\mathrm{e}}(\rho)=0.2 \times 10^{19}\left(1-\rho^{8}\right)^{2} \mathrm{~m}^{-3}$. About $20 \%$ of the injected power is absorbed by high energy electrons at relativistic down shifted second harmonic resonance.

In the case where appreciable amount of high energy electrons exist in the electron distribution function, such high energy electrons can drastically alter the power deposition profile that is the key parameter in the transport analysis. Injected EC wave is absorbed by such high energy electrons and further accelerates these electrons. Such accelerated electrons can make non-diffusive transport and can drastically alter the effective power deposition due to the relativistic downshift of the resonance frequency and through the thermalization process to the bulk electrons. In order to simulate the effect of high energy electrons on ECRH, ray-tracing code (LHDGauss) is extended to accommodate to calculate the multi-Maxwellian electron distribution function. In Fig.3 are shown the power deposition profiles calculated from ray-tracing that includes the high energy electron effects via bi-Maxwellian distribution function. Fig.3a) and b) are local power deposition profile and integrated absorption fraction respectively as a function of the normalized minor radius for the case that the bulk electron temperature is $10 \mathrm{keV}$ without high energy electrons. Fig.3c) and d) are those of the identical bulk electrons but with $10 \%$ high energy density fraction with the averaged energy of $100 \mathrm{keV}$. It was demonstrated that the injected power from the low field side partly absorbed at the relativistically down shifted second harmonic resonances in the low field side by decomposing the absorption fraction to bulk and high energy electrons. This second harmonic absorption amount to about $20 \%$ of injected power in the case where $10 \% 100 \mathrm{keV}$ high energy fraction. Such absorption can work as a screening effect for the central bulk electrons. The relativistic shift of the fundamental resonance is toward the high field side. The broadening of the power deposition profile can not be explained in the case of low field side injection as far as the the absorption by the bulk electrons is dominant at low field side.

A series of experiment is conducted to investigate the acceleration and relaxation process of the high energy electrons. Three gyrotrons, each capable of delivering more than $1 \mathrm{MW}$ power into LHD, are turned on to the low density $\left(0.1 \times 10^{19} \mathrm{~m}^{-3}\right)$ plasma. By changing the injection condition of each antenna and the antenna from which power is modulated, the response of the plasma is observed by diamagnetic stored energy, pulse height analysis of the hard X-ray, and ECE spectra. Figure 4 is the 


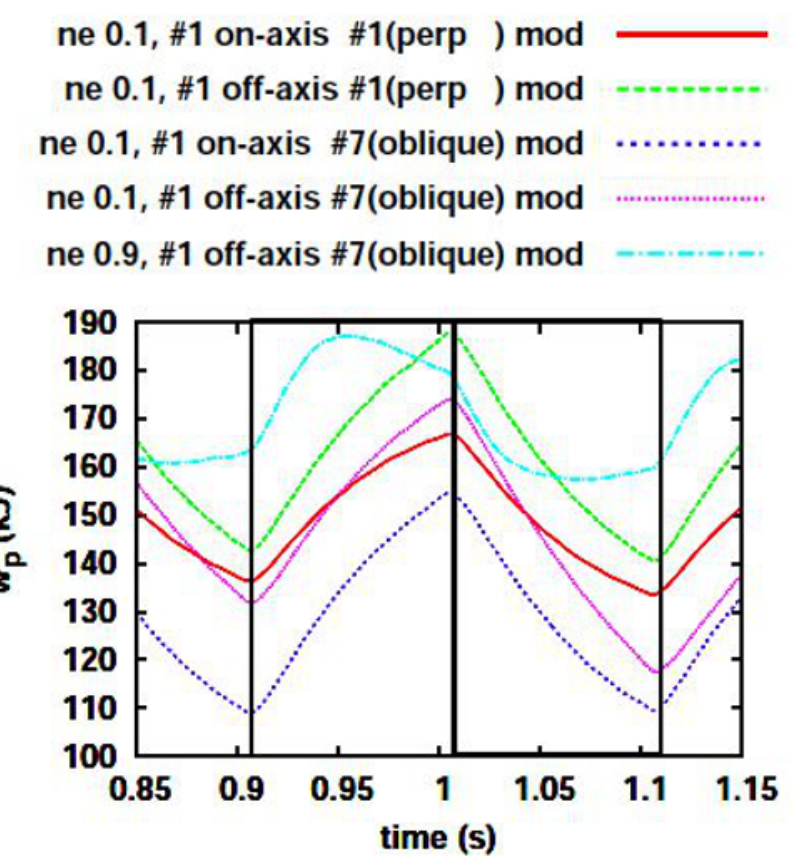

Fig. 4. Time response of the stored energy to $5 \mathrm{~Hz}$ modulated ECH for low density $\left(0.1 \times 10^{19} \mathrm{~m}^{-3}\right)$ cases. The cases for the perpendicular injection beam modulated with on-axis and off-axis are plotted with solid and broken lines, respectively. Dotted lines show the cases where oblique injection beam is modulated for on- and off- axis perpendicular heated plasma with rough and fine dots, respectively. High density case $\left(0.9 \times 10^{19} \mathrm{~m}^{-3}\right)$ with off-axis perpendicular are modulated oblique injection is shown with dashed-dotted line for reference.

example of the response of the stored energy, $w_{\mathrm{p}}$. Here are shown the time response of $w_{\mathrm{p}}$ to the $5 \mathrm{~Hz}$ power modulated ECRH injection for low $\left(0.1 \times 10^{19} \mathrm{~m}^{-3}\right)$. High density $\left(0.9 \times 10^{19} \mathrm{~m}^{-3}\right)$ case is also shown with dashed-dotted line. Maximum $w_{p}$ in low density case reaches to the comparable level as that in the high density case. That is a clear indication of the presence of high energy electrons. The response time of the bulk electrons is the order of 20-50 ms at high density. While that of high energy components is 200 to $300 \mathrm{~ms}$. The more high energy electrons are created and the response of $w_{\mathrm{p}}$ is enhanced as the heating position is shifted off-axis toward high field side. Those are qualitatively explained by the fact that the drift surface of the ripple trapped electrons and the effect of relativistically down shifted resonance.

Macroscopic heating effect can be seen from the time response of the diamagnetic stored energy. In Fig. 4 are shown the time response of the stored energy to the $5 \mathrm{~Hz}$ modulated injection of ECRH power under different injection condition for low density plasmas $\left(0.1 \times 10^{19} \mathrm{~m}^{-3}\right)$ and for a thermal plasma $\left(0.9 \times 10^{19} \mathrm{~m}^{-3}\right)$ as a reference. In the thermal plasma, this response is just $20-30 \mathrm{~ms}$ and well corresponds to the energy confinement time of such plasma parameter range. On the other hand, in low density case where high energy electrons dominate the response time is about 10 times longer and the behavior is non-linear. Such long time constant and non-lineality may be explained by the ECRH power absorption process by high energy electrons and the balance between acceleration and diffusion/thermalization and/or orbit effects. Several injection/modulation combinations of the ECRH are tried to distinguish such effects. Off-axis high field side injection among them had most strong effect on the stored energy. This result suggests that high energy electrons tends to stay in the off axis drift orbits which cross the relativistically down shifted cyclotron resonance at high field side off-axis perpendicular injection angle. 


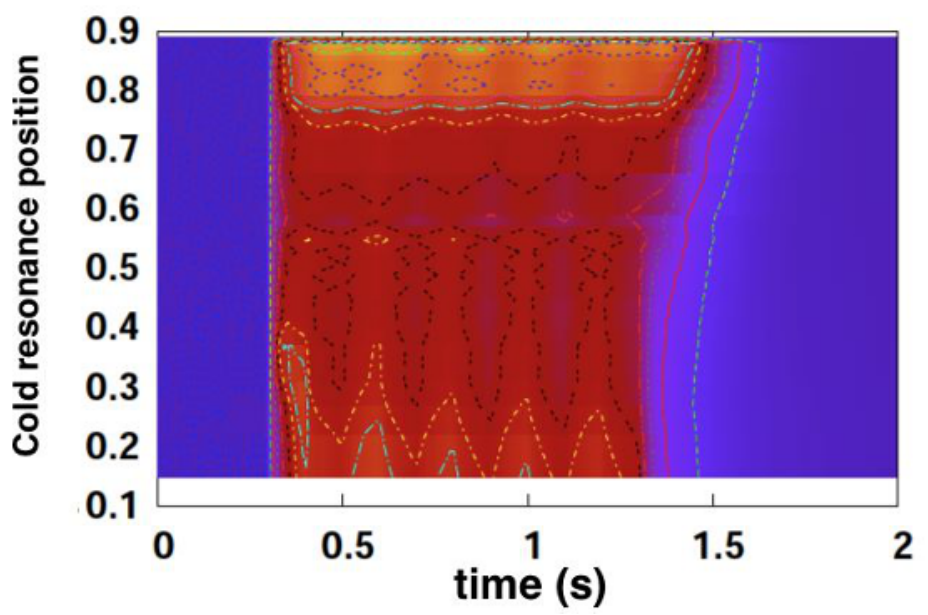

Fig. 5. ECE spectra contour showing the temporal change of the ECE spectrum a function of averaged minor radius corresponding to a second harmonic resonance position. Perpendicular off-axis injection beam is modulated with $5 \mathrm{~Hz}$ on the plasma sustained by obliquely injected ECRH.

ECE spectra gives detailed information about the energy and position of the high energy electrons. In Fig. 5 is shown the contour of the ECE spectra as time in horizontal axis and radial normalized position of the second harmonic cold resonance on the vertical axis. The peaks appear in the low frequency side (upper side corresponding to peripheral second harmonic cold resonance position) shows slower response than the high frequency side. Detailed comparison of the calculated ECE spectra including the high energy components and the results is underway.

\section{Conclusions}

The central bulk electron temperature, $T_{\mathrm{e} 0}$, exceeded $20 \mathrm{keV}$ at averaged electron density, $n_{\mathrm{e}} \leq 3 \times 10^{18}$ $\mathrm{m}^{-3}$. The presence of high energy electrons is confirmed in such low density and high temperature, collision-less plasma. It is demonstrated that the presence of the high energy electrons can drastically alter the power deposition profile that is mainly due to the relativistically down shifted second harmonic resonance at low field side.

\section{Acknowledgement}

This work was supported by National Institute for Fusion Science under NIFS10ULRR501 and 503.

\section{References}

1. H. Takahashi , T. Shimozuma, S. Kubo, S. Ito, S. Kobayashi, Y. Yoshimura, H. Igami, Y. Mizuno, Y. Takita, T. Mutoh, T. Kariya, R. Minami, T. Imai, Fusion Science and Technology, Vol. 57, (2010) 19.

2. M. Yokoyama, K. Ida, H. Sanuki, et al, Nucl. Fusion 42 (2002) 143.

3. S. Kubo et al., J. Plasma Fusion Res. SERIES 5 (2002) 584.

4. N. Marushchenko et al., Plasma and Fusion Research, 2 (2007) S1129.

5. S. Kubo,H. Igami, Y. Nagayama, S. Muto, T. Shimozuma, Y. Yoshimura, H. Takahashi and T. Notake, J.Plasma Fusion Res. Volume 4, (2009) S1095.

6. G. Bekefi, Radiation Processes in Plasma ,John Wiley \& Sons Inc., New York (1966). 